\title{
Gastric adenocarcinoma at the anastomotic site 50 years after gastrojejunostomy: A case report
}

\author{
TSUTOMU NAMIKAWA, YASUHIRO KAWANISHI, KAZUNE FUJISAWA, ERI MUNEKAGE, \\ MASAYA MUNEKAGE, HIROMICHI MAEDA, HIROYUKI KITAGAWA, \\ MICHIYA KOBAYASHI and KAZUHIRO HANAZAKI
}

Department of Surgery, Kochi Medical School, Nankoku, Kochi 783-8505, Japan

Received November 23, 2016; Accepted May 17, 2017

DOI: $10.3892 /$ mco.2017.1309

\begin{abstract}
We herein report a rare case of superficially spreading early gastric cancer occurring 50 years after gastrojejunostomy. An 83-year-old woman was diagnosed with gastric cancer after complaining of epigastric discomfort. The patient had undergone gastrojejunostomy with Braun jejunojejunostomy for benign chronic peptic ulcer 50 years prior. Esophagogastroduodenoscopy revealed an irregular nodular lesion on the gastric side of the anastomosis of the gastrojejunostomy. No abnormal lesions were identified by abdominal contrast-enhanced computed tomography. The patient underwent distal gastrectomy with regional lymphadenectomy. The final diagnosis was signet ring cell carcinoma invading the gastric submucosal layer, without lymph node metastasis, located in the area surrounding the original gastrojejunostomy and measuring $9.5 \times 4.5 \mathrm{~cm}$. In addition, dilated cystic glands were found in the submucosal layer, indicating gastritis cystica profunda. Following surgery, the patient remained symptom-free without evidence of recurrence for 46 months. Although it is not clear whether the adenocarcinoma at the stomal site was associated with the superficial spreading-type tumor in the present case, the observations may provide clues as to the pathogenic process of this entity.
\end{abstract}

\section{Introduction}

Gastric stump cancer following distal gastrectomy is a unique clinical entity with relatively few reported cases, with the development of cancer around the surgical anastomosis considered to be associated with the long-term effects of regurgitation $(1,2)$. We herein report a case of superficially

Correspondence to: $\mathrm{Dr}$ Tsutomu Namikawa, Department of Surgery, Kochi Medical School, Kohasu, Oko-cho, Nankoku, Kochi 783-8505, Japan

E-mail: tsutomun@kochi-u.ac.jp

Key words: gastric adenocarcinoma, gastrojejunostomy, gastric bypass, anastomosis spreading adenocarcinoma near the gastrojejunostomy site, in a background of gastritis cystica profunda, occurring 50 years after gastrojejunostomy for benign chronic peptic ulcer in a patient who had retained the entire stomach.

\section{Case report}

An 83-year-old woman visited her local doctor complaining of epigastric discomfort. Esophagogastroduodenoscopy (EGD) revealed early gastric cancer, diagnosed as adenocarcinoma following histopathological examination of biopsy specimens, and the patient was referred to the Kochi Medical School Hospital in April 2013. The patient had undergone gastrojejunostomy with Braun jejunojejunostomy for benign chronic peptic ulcer 50 years prior. At the time of the most recent presentation, laboratory investigations, including measurement of serum carcinoembryonic antigen and cancer antigen 19-9 levels, revealed no significant abnormalities, apart from a hemoglobin level decreased to $8.5 \mathrm{~g} / \mathrm{dl}$ (normal, 11.6-14.8 g/dl). A second EGD revealed an irregular nodular lesion on the gastric side of the anastomosis of the gastrojejunostomy (Fig. 1). Barium meal examination revealed flow of contrast agent from the stomach to the jejunum through the gastrojejunostomy (Fig. 2). No abnormal lesions were identified by abdominal contrast-enhanced computed tomography.

The patient underwent distal gastrectomy, including the gastrojejunostomy site and the Braun anastomosis, with regional lymphadenectomy, followed by Billroth I reconstruction. Macroscopic examination of the resected specimen confirmed a superficially spreading tumor surrounding the site of the gastrojejunostomy, measuring 9.5x4.5 cm (Fig. 3).

Microscopic examination of the specimen revealed signet ring cell carcinoma and well-differentiated tubular adenocarcinoma invading the gastric submucosal layer, with one lymph node metastasis, but no jejunal invasion (Fig. 4A). There were areas of dilated cystic glands in the deeper parts of the mucosa and submucosa, findings consistent with gastritis cystica profunda (Fig. 4B). The gastritis cystica profunda was distributed in the area around the gastric side of the gastrojejunostomy, extending into the submucosal layers (Fig. 5). The extent of the gastritis cystica profunda was limited to the areas neighboring the anastomosis, and the signet ring cell 


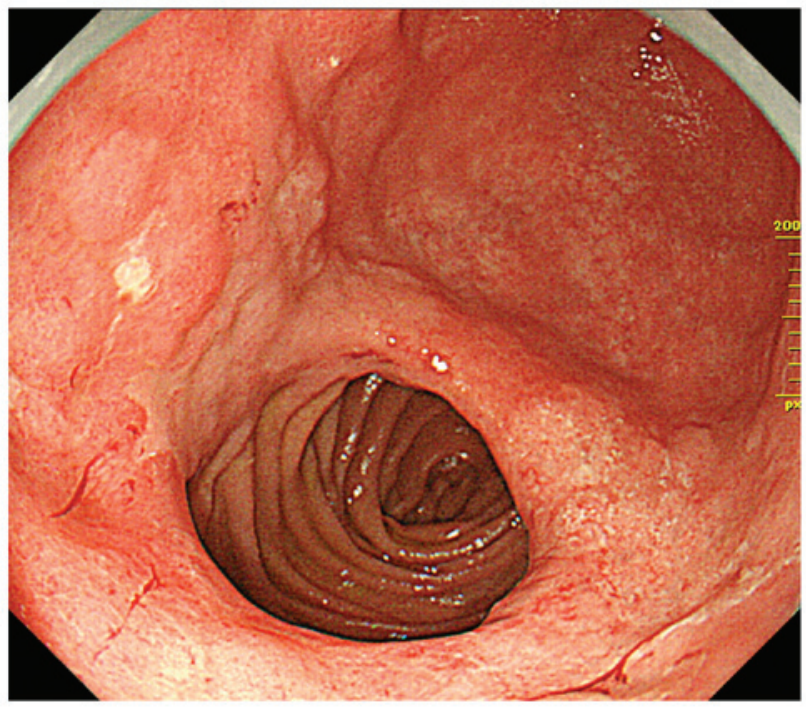

Figure 1. Esophagogastroduodenoscopy showing a superficial, irregularly elevated lesion surrounding the gastrojejunostomy.

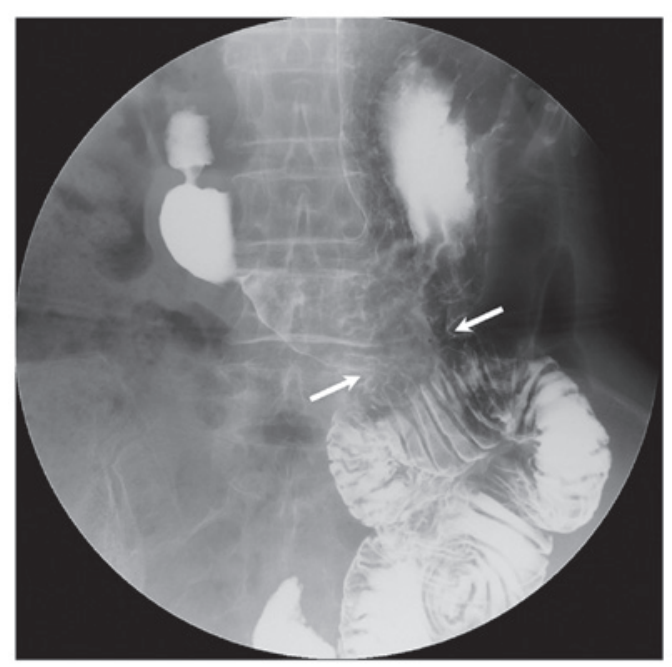

Figure 2. Barium image showing communication between the stomach and jejunum through the gastrojejunostomy (arrows).

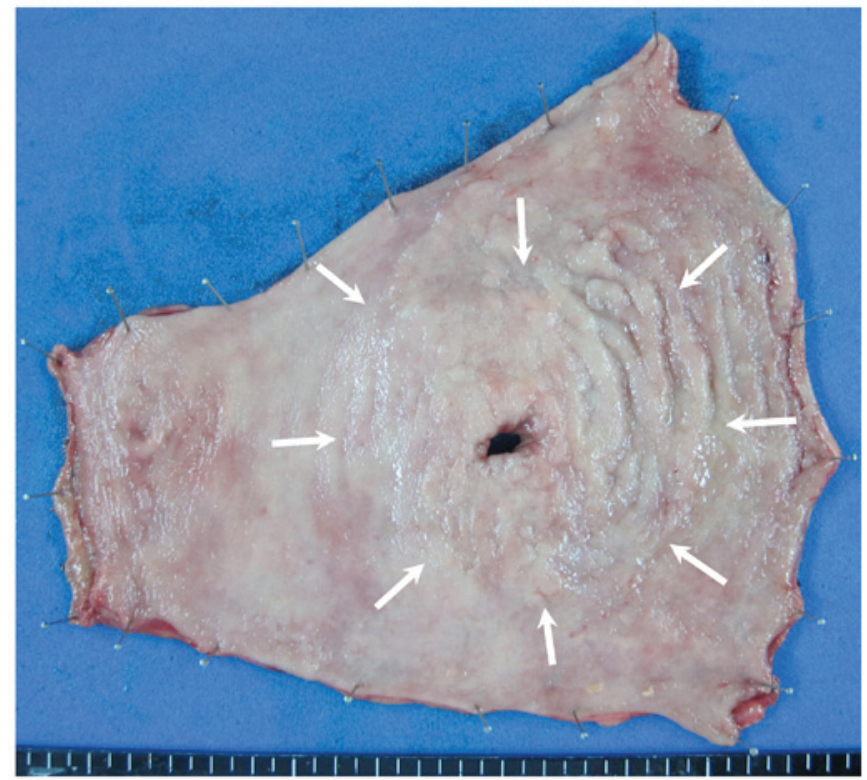

Figure 3. Gross appearance of the surgically resected specimen showing a superficial spreading-type tumor measuring $9.5 \times 4.5 \mathrm{~cm}$ (arrows).

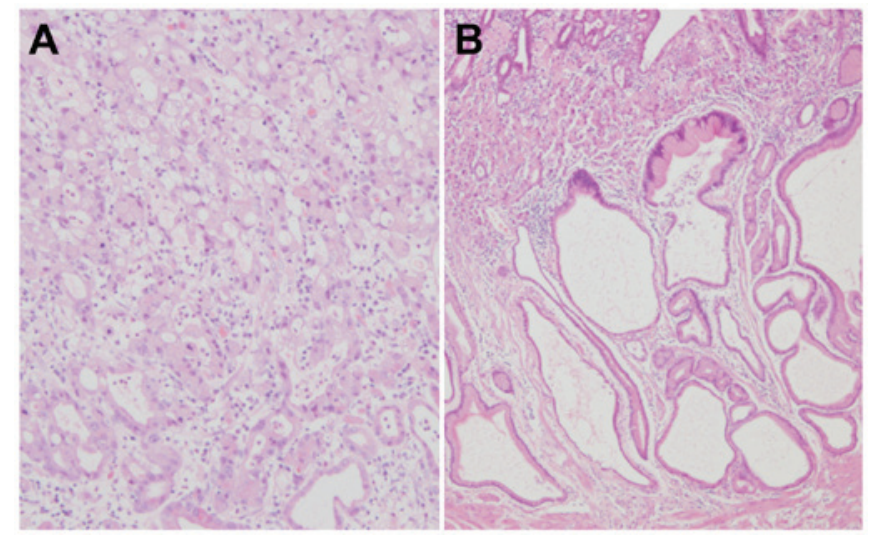

Figure 4. Histological findings of the resected specimen demonstrating (A) signet ring cell carcinoma and (B) dilated cystic glands in the deeper parts of the mucosa and submucosa. Hematoxylin and eosin staining; magnification, (A) $\mathrm{x} 100$ and (B) x40.

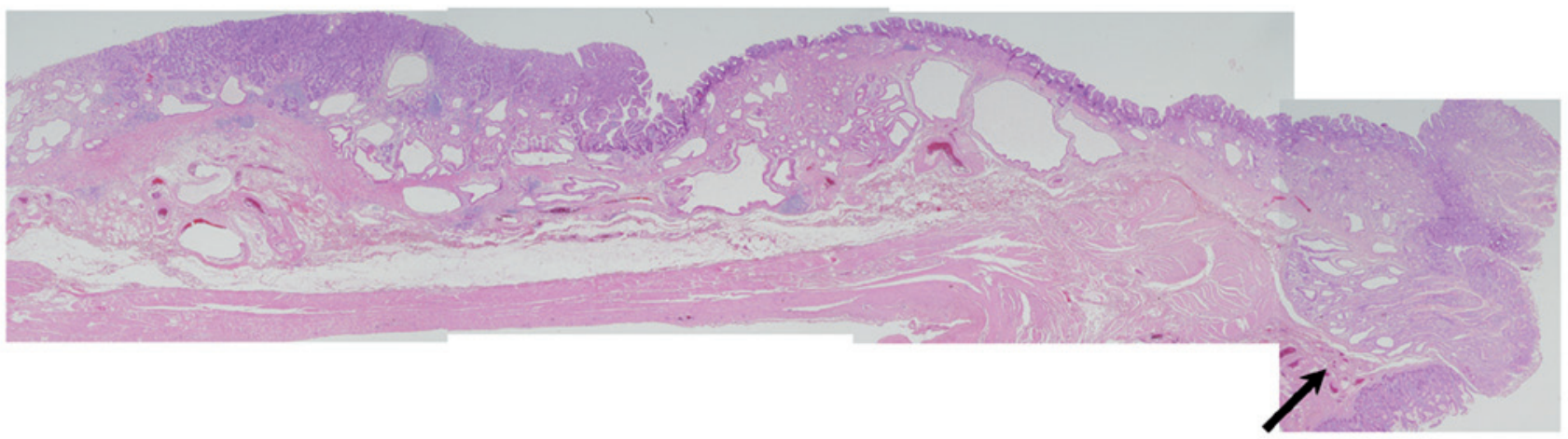

Figure 5. Low-magnification histological image of the area around the gastrojejunostomy showing signet ring cell carcinoma with well-differentiated tubular adenocarcinoma and gastritis cystica profunda. The arrow indicates the edge of the gastrojejunostomy (magnification, $\mathrm{x} 4$ ).

carcinoma and well-differentiated tubular adenocarcinoma (without dilated cystic glands) surrounded this area (Fig. 6).
The immunohistochemical examination using anti-Ki-67 antibody to detect the proliferating cells revealed scattered 


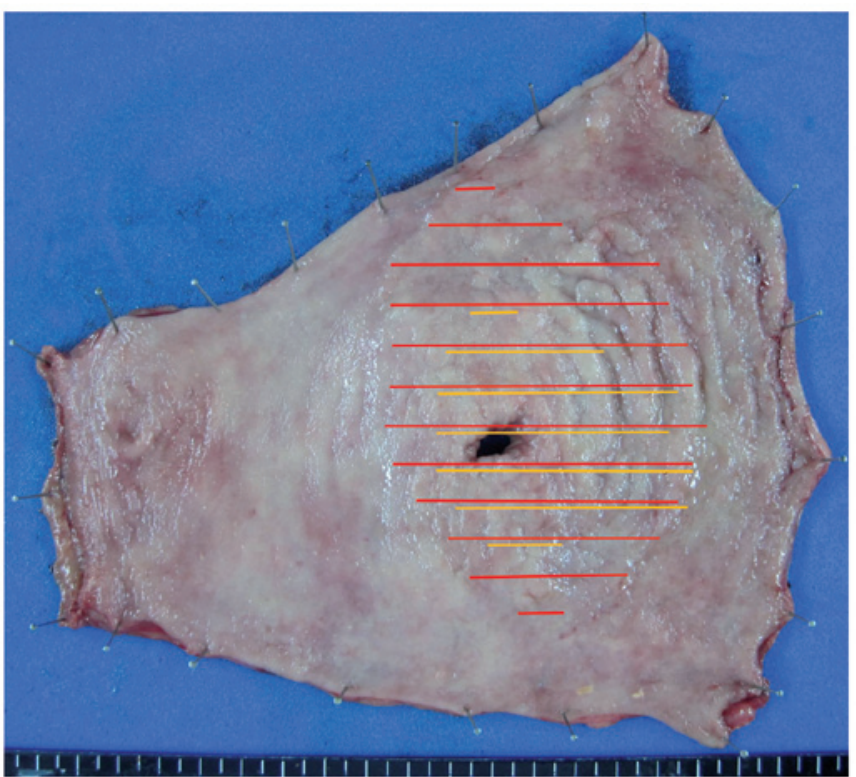

Figure 6. Reconstruction mapping picture showing the distribution of signe ring cell carcinoma with well-differentiated tubular adenocarcinoma (red lines) and the cystic dilated glands (yellow lines), referred to as gastritis cystica profunda.

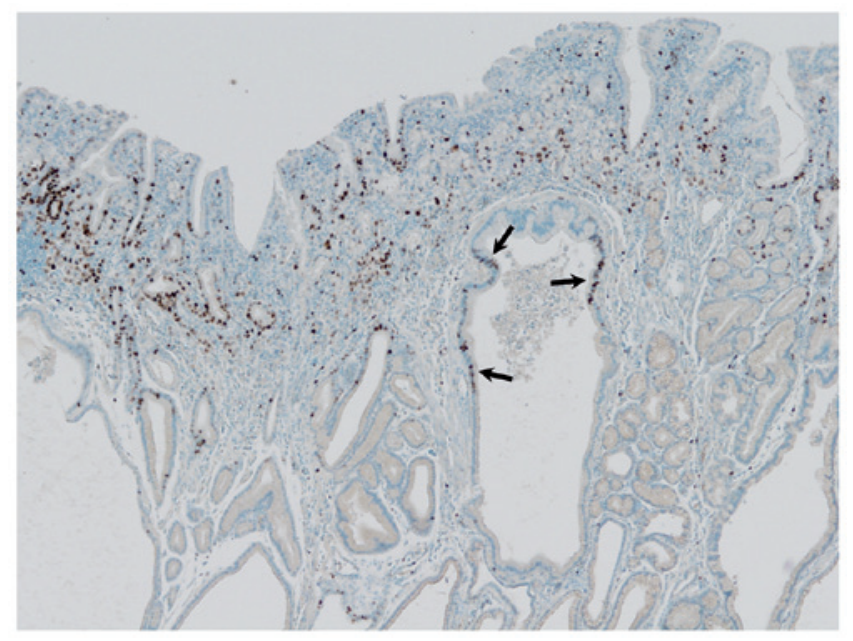

Figure 7. Immunohistochemical examination using anti-Ki-67 antibody for detecting the proliferating cells showing scattered positive staining in the area of gastritis cystica profunda (arrows).

brownish deposits in the area of gastritis cystica profunda, while strong positive staining was observed in the signet ring cell carcinoma (Fig. 7). The postoperative course was uneventful, and the patient underwent periodic follow-up physical examinations. On the last follow-up at 46 months after the operation (April 2017) the patient remained symptom-free, without evidence of recurrence. Written informed consent was obtained from the patient regarding the publication of this case report and associated images.

\section{Discussion}

Gastrojejunostomy may result in later development of gastric cancer. The proposed mechanism for the later development of gastric cancer is irritation of the gastric mucosa by persistent secondary bile reflux and pancreatic secretions, which promote neoplasia (3). Late development of anastomotic carcinoma has been reported, which refers to a time interval of 15-25 years between the initial and second surgeries $(1,2)$. Conversely, only few studies have reported the clinicopathological details of superficially spreading early gastric cancer, as it is a rare disease, and its pathogenesis remains unclear (4). Although it is not clear whether there was an association between the adenocarcinoma at the stomal site and the superficially spreading tumor in the present case, the observations may provide clues as to the pathogenic process underlying this disease entity.

Gastritis cystica profunda, a benign disease characterized by cystic dilatation of the gastric glands that extend into the submucosa of the stomach, develops on the gastric side of the anastomosis in patients who have undergone gastrectomy (3). It has been suggested that mucosal injury caused by surgical procedures or the suturing technique itself may promote mucosal prolapse and herniation of glands into the submucosa, which are also considered as potentially precancerous lesions $(3,5)$. The results of the present case following immunohistochemical examination using Ki-67 demonstrated low proliferative activity of the cells in the area of gastritis cystica profunda, which may indicate that it is a para-cancerous lesion. Of note, in the present case, the gastric cancer near the site of the original anastomosis developed 50 years after the original surgery. Further studies and assessments by accumulating additional cases are required to gain an understanding of the various presentations of this entity.

\section{References}

1. Namikawa T, Kitagawa H, Iwabu J, Okabayashi T, Kobayashi M and Hanazaki K: Tumors arising at previous anastomotic site may have poor prognosis in patients with gastric stump cancer following gastrectomy. J Gastrointest Surg 14: 1923-1930, 2010.

2. Tanigawa N, Nomura E, Niki M, Shinohara H, Nishiguchi K, Okuzawa M, Toyoda M and Morita S: Clinical study to identify specific characteristics of cancer newly developed in the remnant stomach. Gastric Cancer 5: 23-28, 2002.

3. Mukaisho K, Miwa K, Kumagai H, Bamba M, Sugihara H and Hattori T: Gastric carcinogenesis by duodenal reflux through gut regenerative cell lineage. Dig Dis Sci 48: 2153-2158, 2003.

4. Namikawa T, Kitagawa H, Iwabu J, Okabayashi T, Sugimoto T, Kobayashi M and Hanazaki K: Clinicopathological properties of the superficial spreading type early gastric cancer. J Gastrointest Surg 14: 52-57, 2010.

5. Lee TH, Lee JS and Jin SY: Gastritis cystica profunda with a long stalk. Gastrointest Endosc 77: 821-822, 2013. 